\title{
Normal and malignant hematopoiesis
}

\author{
M. F. Fey \\ Institute of Medical Oncology, Inselspital, Bern, Switzerland
}

\section{hematopoiesis and stem cells or progenitor cells}

Hematopoiesis is a delicately regulated process, which results in the production of various blood cell types with functions ranging widely from oxygen transport to immunity. The maintenance of this system requires hematopoietic stem cells (HSC) in the bone marrow that give rise to committed progenitor cells, which in turn proliferate and differentiate into functional end cells. Stem cells were initially discovered as cells generating hematopoietic colonies in the spleen of lethally irradiated mice, and hence the term 'colony-forming units (CFU)' with the affix ' $S$ ' coined for 'spleen'. Later, progenitor cells producing CFU-S were found to be distinct from more primitive bone marrow cells termed HSC. HSC are adult stem cells, able to undergo self-renewal, or apoptosis, and to generate progenitor cells for all hematopoietic lineages on demand. HSC seem to maintain some stem-cell plasticity in vitro, implying that under particular conditions they may differentiate into nonhematopoietic cell lineages. Whether this mechanism is important under physiological in vivo conditions is unclear. The self-renewal capacity of HSC is associated with telomerase activity, a reverse transcriptase preventing chromosomal telomere shortening during cell division (which otherwise would lead to genetic instability and replicative senescence). As HSC divide, they first form common lymphoid and common myeloid progenitors, which further diversify and differentiate to $\mathrm{B}, \mathrm{T}$, or natural killer (NK) cells in the lymphoid lineage or to granulocyte/monocyte, megakaryocyte, and erythrocyte precursors within the myeloid lineage. Human HSC are characterized by typical protein expression patterns. They are notably CD34+ (CD34 is a cell adhesion molecule) and CD38-. CD34+ cells derived from peripheral blood, bone marrow, or umbilical cord blood are able to provide long-term engraftment of hematopoiesis in the bone marrow. Peripheral blood CD34+ HSC have become the most frequently used source of stem cells in allogeneic or autologous stem-cell transplantation, providing faster neutrophil and platelet recovery than bone marrowderived CD34+ HSC. Transplantation using stem cells derived from umbilical cord blood is possibly effective, but still experimental and limited by the modest cell doses available from such harvests (see Figure 1).

Hematopoiesis also depends on the microenvironment offered within the bone marrow cavities. HSC and their progeny are embedded in a meshwork of nonhematopoietic cells, such as fibroblasts, osteoblasts, and adipocytes, which in addition to providing mechanical anchorage are also the source of factors essential to sustain successful hematopoiesis. For example, fibroblasts are an important source of granulocyte-macrophage colony-stimulating factor (GM-CSF). Endothelial cells in the marrow express CD34 which provides a homing signal to HSC to remain there, and also antagonizes their maturation. Disorders affecting nonhematopoietic cells may lead to failure of hematopoiesis, as e.g. in osteomyelofibrosis, where marrow cavities are obliterated by dense fibrous tissue, and hematopoiesis is exiled to other organs such as the liver or the spleen.

\section{myeloid lineages}

As a first step towards the myeloid lineage of hematopoietic cell differentiation, HSC produce the common myeloid precursor cell [producing cells of all myeloid lineages, hence labeled CFUGEMM (for CFU-granulocyte-erythrocyte-macrophagemegakaryocyte)] which gains additional markers present on CD34+ HSC. CD33 expressed on CFU-GEMM has become of clinical interest, since anti-CD33 antibodies are now being tested in clinical trials to treat acute myelogenous leukemia (AML) expressing this protein. CFU-GEMM next move on to feed the different myeloid lineages, i.e. erythroid, granulocyte/ monocyte, and megakaryocyte production lines, where cells become increasingly specialized in their functions. These cellular differentiation programs are largely maintained in clonal neoplastic myeloproliferative disorders [e.g. chronic myelogenous leukemia (CML)], but AML typically show deficient maturation, and hence AML blasts are arrested early in the myeloid production line.

Erythropoiesis shows two classes of progenitors. Burstforming units (erythroid) (BFU-E) are the earliest progenitors committed exclusively to erythroid differentiation, and they give rise to multi-clustered colonies of hemoglobin-producing cells. The second more differentiated class consists of colony-forming units (erythroid) (CFU-E). Erythropoietin is a compulsory growth factor for erythroid development at the BFU-E and CFU-E level, but it does not play a role in determining lineage commitment of HSC. As myeloid progenitor cells move into the granulocyte/monocyte lineage, they acquire characteristic markers such as myeloperoxidase or CD38 which is expressed on myeloblasts, but they lose 'early' markers such as CD33 which is not present beyond the myelocyte stage. A number of 'late' markers such as CD11b or leukocyte alkaline phosphatase are still present on mature granulocytes. Interestingly, 


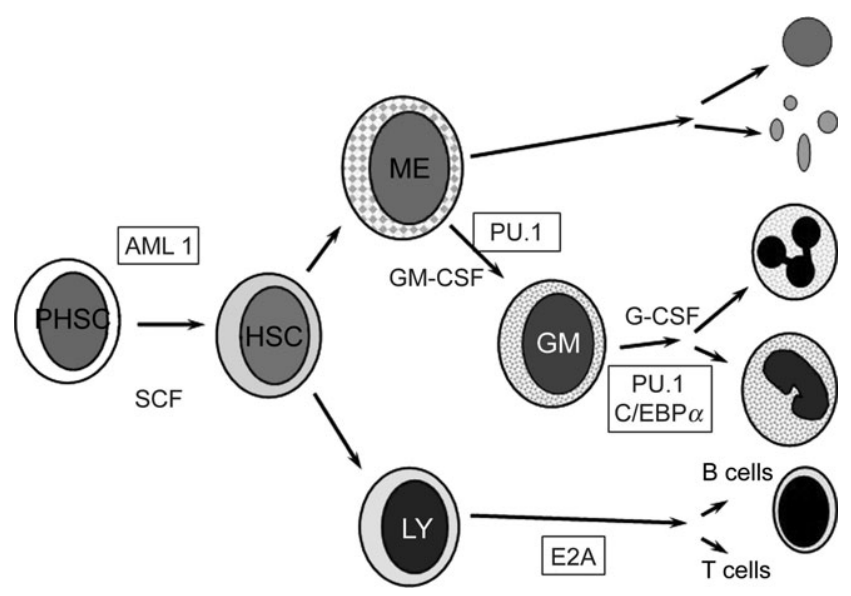

Figure 1. Hierarchical model of haematopoiesis. Pluripotent haematopoietic stem cells (PHSC) feed into various haematopoietic lineages whilst retaining the capacity for self-renewal. Lineage-committed stem cells produce progeny destined to differentiate into red cells, granulocytes, lymphoid cells, platelets etc. Proliferation and differentiation in the bone marrow are directed by a concerted action of growth factors (SCF, GM-CSF or G-CSF) and transcription factors which are particularly important for cellular differentiation (i.e., myeloid: $\operatorname{PU1}, \mathrm{C} / \mathrm{EBP}(\alpha)$; lymphoid: E2A).

granulocytes in CML that are part of the malignant neoplastic clone are leukocyte alkaline phosphatase negative. Granulocytic precursors acquire typical granules. Primary granules are present as of the promyelocytic stage [hence, acute promyelocytic leukemia (APL) blasts are often heavily granulated], and secondary granules are acquired at the transition to the myelocyte stage. They contain proteins that are only seen in cells of granulocytic lineage, and are important for their proper functioning in host defense. AML are clonally derived from transformed stem cells with the capacity to differentiate up to an early point in myelopoiesis. For example, blasts in APL reach the promyelocytic stage, but do not differentiate beyond (maturation arrest).

Monocyte ontogeny follows the same principles as explained for the granulocytic lineage. Monocytes may in turn serve as precursors for dendritic cells, which are professional antigenpresenting cells (APC) (see later). Unique cell-surface markers indicating monocytic differentiation have been difficult to identify. CD11b is also present on cells of the monocytic lineage, if not specific, but CD14, a lipopolysaccharide receptor, is major functional surface protein on monocytes.

Megakaryopoiesis and ultimately platelet formation follow the same principles. Progenitor cells committed to the megakaryocyte lineage differentiate into promegakaryoblasts, which in turn form mature megakaryocytes. These latter cells, very conspicuous in bone marrow smears are unable to undergo further cell division, but retain the capacity for DNA synthesis and thus become polyploid. Platelets are shed from megakaryocytes as 'blebs' formed of their cytoplasm. Thrombopoietin is a chief stimulatory player in thrombopoiesis, operating at all levels from progenitor cells to mature megakaryocytes.
The identification of soluble factors responsible for the extrinsic regulation of hematopoiesis had to await the era of molecular biology to identify them as cytokines modulating proliferation, differentiation, and the function of hematopoietic cells. The cytokine family chiefly includes the CSF, which stimulate CFU activity of hematopoietic progenitor cells. Examples of CSF are erythropoietin or granulocyte colonystimulating factor (G-CSF). Erythropoietin is a typical hormone produced in the kidney and released into the circulation to reach its target cells in the bone marrow, particularly in response to hypoxia. G-CSF induces the proliferation of granulocytic progenitors and their differentiation to mature granulocytes and it increases the functional activity of mature neutrophils (such as chemotaxis, phagocytosis, and the like). Recombinant G-CSF is clinically used to mobilize peripheral blood stem cells, which it achieves by stimulating matrix metalloproteinase release from degranulated neutrophils. These enzymes cleave adhesion molecules expressed in bone marrow stromal cells, which in turn causes the release of CD34+ stem cells into the circulation. G-CSF mobilization of stem cells keeps them in the $G_{0} / G_{1}$ phase of the cell cycle. Recombinant GM-CSF is no longer used in the clinic, except in experimental immunotherapy protocols with the aim to recruit dendritic APC.

The intrinsic regulation of hematopoiesis was overshadowed in the literature by the excitement about the cloning of growth factor genes. The role of hematopoietic transcription factors was, however, increasingly gleaned from the study of the molecular pathology of human acute leukemias. Many chromosomal translocations in acute leukemias affect transcription factors and profoundly alter their expression, leading to aberrant cellular differentiation or apoptosis programs. The term 'co-stimulatory cytokines' is sometimes used for such molecules, which show little or no direct effect on colony formation, but have the capacity to act synergistically with CSF. Co-stimulatory cytokines include stem-cell factor (SCF or c-kit ligand) or the FLT3 tyrosine kinase. SCF is a transcription factor, which reacts with the c-kit receptor present on CD34+CD38- bone marrow cells. It accelerates their entry into the cell cycle, and in cooperation with BCL-2 prevents apoptosis. SCF influences the differentiation to all hematopoietic lineages. It may collaborate with almost every known hematopoietic cytokine to promote colony formation. Involvement of SCF in chromosomal translocations leads to its inappropriate expression and thus contributes to leukemogenesis, particularly in T-cell acute lymphoblastic leukemia (ALL). Aberrant expression of FLT3 (often caused by internal tandem duplications within the gene) is the single most common gene abnormality in AML, and associated with an aggressive clinical course. In contrast to such 'general practitioners' in hematopoiesis, master regulators of hematopoietic lineage commitment are specialized to a particular lineage. For example, the CCAAT enhancer-binding protein alpha (C/EBP alpha) is essential for normal neutrophil granulocyte formation. Mutations inactivating the C/EBP alpha gene lead to an AML subtype with a relatively good prognosis. The molecular diagnosis of FLT3 and C/EBP alpha gene mutations has provided important prognostic factors of clinical relevance in AML, particularly within the heterogeneous group of AML with a normal karyotype. 


\section{the immune system and lymphoid lineages}

The human immune system is assigned the seemingly impossible task to keep at bay pathogens seeking to invade the organism, to clear away dead cells and tissues, to tell the organism's own cells from foreign cells (such as those introduced through organ transplantation), and to assist in wound healing. Conceptually, it may be divided into the innate system and the adaptive system.

The innate immune system encompasses granulocytes, monocytes, NK cells, and related cells as effector cells, which typically act with a very short response time of within hours to a few days. Pathogen recognition receptors (PRP) capable of binding to specific pathogen-associated molecular patterns (PAMP) may be present on their surface or as soluble proteins in the circulation. The C-reactive proteins, often used in the clinic as markers for the presence and activity of infection, are an example. Typically cells operating in the innate system are released as fully mature and functional cells from the bone marrow. Consequences of PRP-PAMP interaction are the phagocytosis of microorganisms, and synthesis as well as release of interferons (IFNs), interleukins (ILs), chemokines, and hematopoietic growth factors, many of which serve to amplify the defense mechanisms against any aliens seeking to invade the host.

The adaptive immune system deals primarily with the generation of T-cell receptor (TCR) and B-cell receptor [i.e. immunoglobulin (Ig)] diversity. It thus achieves two goals, which are not met by the innate system: (i) the generation of an incredibly diverse receptor repertoire and (ii) the amplification of pathogen-specific cells as a reaction to the organism's exposure to pathogen. Innate immune function entirely depends on molecules encoded by genes in germline configuration, whereas the biological diversity of the adaptive system and its specific immune responses are basedon the creation of genetic diversity through physiological antigen receptor gene rearrangements and somatic mutations, which profoundly change the structures of germline TCR and Ig genes. The cells contributing to adaptive immunity are the lymphoid cells, with their production line determined very early in the hematopoietic differentiation process, where the common myeloid precursor splits from the common lymphoid precursor cell.

\section{B cells}

B cells are the subset of lymphocytes specialized to synthesize and release Ig. They are generated in the bone marrow, the primary B-cell production site, from common lymphoid progenitor cells which are $\mathrm{CD} 34+$, but lineage negative $\left(\operatorname{lin}^{-}\right)$, implying that they lack expression of determinants present on mature myeloid and mature lymphoid cells. Immature B cells next move to the spleen where they differentiate into mature but still naive B cells in a process referred to as secondary B-cell development. The marginal zone corresponds to the outer limit of the splenic white pulp and plays a critical role in the immune response to T-cell-independent antigens. The maturation of mature naive $\mathrm{B}$ cells depends on their positive selection by antigen, or else they will die by neglect. Mature naive B cells next migrate to lymph nodes where they undergo further differentiation to become highly specialized effector plasma cells producing specific antibody $(\mathrm{Ab})$ in response to antigenic challenge. In the secondary lymphoid organs, and upon antigenic stimulation, they proliferate and differentiate further. Nonresponsive B cells accumulate in the mantle zone of follicles, surrounding the proliferating antigen-responsive B cells present in a central germinal center of the follicles. Here they are exposed to antigens presented by follicular dendritic cells rather than in a soluble form, and develop via morphologically defined stages of centroblasts or centrocytes to become either Igsecreting plasma cells or memory B cells. Malignant lymphoid neoplasms mirror these various stages of lymphoid ontogeny which is reflected by their morphology and their immunological and molecular profiles. For example, follicular lymphoma and diffuse large-B-cell lymphomas are derived from malignant transformed B cells that show characteristics of germinal center B cells, whereas the neoplastic cells in myeloma produce and often secrete monoclonal Ig proteins, or at least parts thereof (e.g. Ig light chains or Bence-Jones protein).

Critical checkpoints in this line of events are the transition from pro- $\mathrm{B}$ cells to pre-B cells, still in the bone marrow, and the move of transitional B cells to the spleen, at the point when they start to express Ig light chains. The B-cell development pathway is marked by the transient and stage-specific expression of a number of cell-surface-associated molecules, which can be detected by fluorescence-associated cell sorting (FACS) and have become important in the classification of B-cell lymphoid neoplasias. For example, early lymphoid precursors express CD34+ and CD10, which in pro-B cells is complemented by the B-cell markers CD19 and CD20. The physiological function of CD20 is unknown, but nevertheless, CD20 has become important in clinical oncology as the target of a monoclonal antibody, rituximab, that has become established as a complement to chemotherapy in a variety of B-cell lymphomas and leukemias expressing this molecule. As B cells become capable of expressing Ig heavy-chain genes of the $\mu$ type, CD34+ expression is lost, as is CD10 expression at the point where B cells start to express surface Ig light chains and move to the spleen. ALL can occur through neoplastic transformation of early B-lymphoid cells in the bone marrow. Similar to AML, ALL cells, e.g. B-cell precursor ALL blasts, are arrested at a particular stage of B-lymphoid differentiation and retain the respective marker profile.

B-cell-committed precursors and their downstream progeny show rearrangement of Ig genes as a crucial feature of normal Bcell development, since it forms the molecular basis to generate antibody diversity. The earliest B-lineage-committed progenitor is the pro-B cell where Ig heavy-chain gene rearrangement is initiated. Ig heavy-chain genes are composed in the germline configuration of a number of distinct separate sections termed the variable $(\mathrm{VH})$, diversity $(\mathrm{DH})$, joining $(\mathrm{JH})$, and constant (C) regions. In a first step, a DH region is singled out and placed next to a $\mathrm{JH}$ region, whereafter a $\mathrm{VH}$ region rearranges to the $\mathrm{D}-$ JH complex (VHDJH joining). This process much depends on enzymic machinery, where recombinases and recombinaseactivating genes act through the recognition of recombination signal sequences located near the $\mathrm{VH}, \mathrm{JH}$, or D regions. Since 
such signal sequences also occur near oncogenes, e.g. the BCL-2 oncogene, recombinases may by mistake incorporate such alien genes in the Ig gene recombination process, which provides a molecular mechanism for the creation of chromosomal translocations in B-cell neoplasias. If Ig heavy-chain gene rearrangement is successful, cells firstly express the Ig heavy chain of the mu-class, and are at this stage referred to as pre-B cells. Rearrangement of Ig light chains is next in line, always starting with kappa gene rearrangement and passing on to the lambda gene, if kappa rearrangement fails. In parallel, heavychain production may switch from mu-chains to gamma chains present in immunoglobulin G. Once Ig light-chain rearrangement has occurred, pre- $\mathrm{B}$ cells mature into $\mathrm{B}$ lymphocytes that express the newly assembled Ig molecule on their surface. Genetic diversity in the Ig gene repertoire can be further enhanced through the introduction of extra nucleotides (not encoded in the germline Ig gene) to DJH and VH-DJH junctions by a nuclear enzyme termed terminal deoxynucleotidyl transferase (TdT). TdT expression is regularly assessed in FACS-generated marker profiles in lymphoid neoplasias, and its presence is restricted to immature lymphoid cancers, particularly B-cell ALL. Finally, somatic mutations can occur in the $\mathrm{V}$ regions of a rearranged Ig gene, usually in secondary lymphoid organs, and thus lead to even further diversity and an increased affinity of the antibody to antigen. Assessing the structure of antigen receptor genes has become an important tool in molecular diagnostics of B-cell

lymphoproliferations. Since Ig gene rearrangement creates gene sequences that are unique to a given B cell and its progeny, the configuration of Ig genes has become an important marker of clonality. In clonal neoplastic B-cell proliferations, all cells share the very same Ig gene rearrangement, which can be detected on molecular analysis, e.g. as a distinct single amplification fragment produced by an Ig gene-specific PCR, whereas in polyclonal lymphoid proliferations (seen in inflammatory conditions) many different Ig gene fragments would be amplified. Leukemia-specific Ig gene rearrangements can specifically be detected by highly sensitive PCR and thus singled out in clinical diagnostic material to trace minimal residual leukemia after treatment, e.g. in ALL the presence or absence of somatic mutations in the $\mathrm{VH}$ regions of rearranged Ig genes has become important in the classification of B-cell chronic lymphocytic leukemia (B-CLL), since B-CLL exhibiting somatic VH mutations fare much better than B-CLL without this molecular feature.

Specification towards a B-cell lineage is governed by a number of genes, e.g. the transcription factors E2A and PAX5. Both genes can be involved in chromosomal translocations, with their aberrant expression contributing to the development of malignant B-cell neoplasia. E2A is a partner gene in the translocation $t(1 ; 19)$ seen in B-cell precursor ALL and in pre$\mathrm{B}$-cell ALL, whereas PAX5 is involved in translocations found in $\mathrm{Ki}^{+} \mathrm{B}$-cell lymphoma.

\section{T cells}

Similar principles as in B cells mostly apply to the maturation of $\mathrm{T}$ cells, however, with a number of important differences. The common lymphoid progenitor cells in the bone marrow send out their progeny destined to form $\mathrm{T}$ cells to the thymus which is critical for further development and final commitment to the T-cell lineage. Within a day or so of their entry, thymic lymphoid progenitors express CD25 (i.e. the IL-2 receptor). Pro-T cells still maintain their TCR genes in the germline, but TCR-beta-chain rearrangement begins with their move to become pre-T cells. The molecular diagnosis of TCR gene rearrangements can be used to assess clonality of T-cell lymphoproliferations, which is often helpful, since there are no easily detectable immunological surface markers available for this particular purpose. The molecular machinery to achieve TCR gene rearrangements in T cells is very similar to the one used for Ig genes in B cells, and likewise, mistakes may lead to chromosomal translocations. As in B-cell neoplasia, these usually involve a TCR gene on one chromosome, and an oncogene on the partner chromosome. Further along the T-cell differentiation line, pre-T cells must undergo commitment to become either CD4+ helper cells or the CD8+ cytotoxic T cells. This step is usually prompted by the interaction of their TCR protein with peptide/major histocompatibility complexes (MHC). MHC class I molecules induce differentiation towards CD8+ cytotoxic T cells, whereas MHC class II are associated with CD4+ helper T-cell formation. The T-cell immune response, part of the adaptive immune response system, is hence triggered by contact with antigen peptide/MHC complexes. Intracellular or endogenous antigens such as viral proteins in the cytoplasm are broken down by proteosomes, transported to the endocytoplasmic reticulum, and associated with MHC class I molecules to be presented to the immunocompetent cells. Extracellular or exogenous antigens are taken up by specialized APC, dendritic cells, or macrophages by endocytosis; broken down into peptides in lysosomes; and associated with MHC class II molecules. After contact with peptide/MHC class II complexes on APC, CD4+ helper $\mathrm{T}$ cells initiate specific immune responses by secreting first of all soluble proteins such as IL-2 or IFN- $\gamma$. These cytokines provide the required assistance for the activation and differentiation of other lymphoid cells (which are thus 'helped' to develop as required). CD4+ $\mathrm{T}$ cells also regulate immune responses through direct cell-cell contact and not only via secreted factors. If $\mathrm{T}$ cells expressing Fas, a member of the tumor necrosis factor receptor family, meet other cells expressing Fas ligand (FasL) on their surface, $\mathrm{Fas}^{+} \mathrm{T}$ cells will undergo apoptosis. Without such a mechanism of T-cell elimination, the immune compartment would soon be overpopulated. Unfortunately, some tumors also express FasL which provides them with an escape mechanism from immune surveillance by activated tumor-specific T cells. CD8+ cytotoxic $\mathrm{T}$ cells (or cytotoxic T lymphocytes) may produce cytokines upon contact with APC and kill the messenger that bears the peptide/MHC class I complex for which they are specific. Similar to the B-lymphoid system, T-cell neoplasms mirror various stages of T-cell maturation, with T-cell ALL corresponding to the early $\mathrm{T}$-cell progenitor stage, and peripheral T-cell lymphomas exhibiting markers of their more mature postthymic normal counterparts. For example, mycosis fungoides is a neoplasm clonally derived from transformed CD4+ helper T cells. 


\section{summary}

In summary, our knowledge on the physiology of hematopoiesis and the immune system has provided much specific information of direct clinical relevance. The cloning of hematopoietic growth factors or hormones has produced compounds (such as erythropoietin or G-CSF) that help to overcome side effects of chemotherapy, and provide convenient techniques to harvest hematopoietic progenitor cells for transplantation. The study of human leukemias has elucidated the roles of genes important both in normal and leukaemic hematopoiesis. Thus, molecular leukemia markers have become available that are of unique clinical significance to assess prognosis, to predict appropriate therapy, and to follow patients after treatment detecting minimal residual disease. Finally, the molecular unraveling of the unique molecular aberration in CML has provided specific drugs, i.e. ABL tyrosine kinase inhibitors, to induce a return of the marrow to assume normal hematopoiesis. Along that way, molecularly targeted drugs to overcome the differentiation block in acute leukemias are on the horizon, to complement if perhaps not to replace cytotoxic chemotherapy. 